\title{
Article \\ Optimization Design of Step Stress Accelerated Degradation Test for Motorized Spindle Based on $D_{s}$-Optimality
}

\author{
Hongxun Zhao ${ }^{1,2}$, Zhaojun Yang ${ }^{1,2}$, Chuanhai Chen ${ }^{1,2, *}$, Hailong Tian ${ }^{1,2} \mathbb{1}$, Xiaoxu Li $^{3}$ and Shizheng Li ${ }^{1,2}$ \\ 1 Key Laboratory of CNC Equipment Reliability, Ministry of Education, Jilin University, \\ Changchun 130025, China; hxzhao16@mails.jlu.edu.cn (H.Z.); yjz@jlu.edu.cn (Z.Y.); tianhl@jlu.edu.cn (H.T.); \\ 1sz18@mails.jlu.edu.cn (S.L.) \\ 2 School of Mechanical and Aerospace Engineering, Jilin University, Changchun 130025, China \\ 3 School of Mechanical and Vehicle Engineering, Changchun University, Changchun 130025, China; \\ 1xx13313@gmail.com \\ * Correspondence: chen49411@gmail.com
}

Citation: Zhao, H.; Yang, Z.; Chen,

C.; Tian, H.; Li, X.; Li, S. Optimization Design of Step Stress Accelerated Degradation Test for Motorized Spindle Based on $D_{s}$-Optimality. Appl. Sci. 2021, 11, 3832. https:// doi.org/10.3390/app11093832

Academic Editor:

Giangiacomo Minak

Received: 18 March 2021

Accepted: 22 April 2021

Published: 23 April 2021

Publisher's Note: MDPI stays neutral with regard to jurisdictional claims in published maps and institutional affiliations.

Copyright: () 2021 by the authors. Licensee MDPI, Basel, Switzerland. This article is an open access article distributed under the terms and conditions of the Creative Commons Attribution (CC BY) license (https:// creativecommons.org/licenses/by/ $4.0 /)$.

\begin{abstract}
In the accelerated degradation test (ADT) of motorized spindles, it is necessary to apply a variety of stresses to simulate real working conditions. However, the traditional accelerated test scheme optimization method does not consider the weight of various stresses in the test, resulting in the evaluation accuracy of important stress parameters in the model being too low. In order to solve this problem, an optimal design method of the step stress accelerated degradation test (SSADT) scheme for motorized spindles is proposed based on $D_{s}$-optimality. Firstly, the fault tree analysis (FTA) method is used to analyze the collected fault data of motorized spindles and screen the main stress. Then, the accelerated degradation model is established by using drift Brownian motion. Based on the $D_{s}$-optimality, the optimization variables and constraints in the test are determined, and the optimization model is established with the objective of minimizing the estimated variance of the main stress parameters in the acceleration model; additionally, the optimization steps are given. Finally, an example is given to verify the effectiveness of the method. Sensitivity analysis of the optimization results shows that the method has good robustness.
\end{abstract}

Keywords: motorized spindle; step stress accelerated degradation test; test analysis; $D_{\mathcal{S}}$-optimality; optimization design

\section{Introduction}

As one of the key functional components of computer numerical control (CNC) machine tools, the reliability of motorized spindles has an important impact on the reliability of CNC machine tools [1]. For complex electromechanical and hydraulic products, such as motorized spindles, a reliability test is the only way to verify the reliability level of a motorized spindle and expose its weak links. Because the reliability level of a motorized spindle is high, it is usually necessary to carry out accelerated tests to evaluate its reliability. However, the traditional accelerated life test (ALT) [2] aims to obtain the failure data, and then evaluate the reliability of the product, and improve the reliability design according to the evaluation results, so as to improve the reliability of the product. However, with the development of science and technology, the reliability level of motorized spindles is constantly improving. This traditional accelerated life test method has been unable to obtain enough fault data of motorized spindle in a short time, and then cannot obtain an accurate reliability level for motorized spindles. ADT can product failure or performance degradation in a short time by applying higher-than-normal stress in the test, and evaluate the reliability of the product under normal stress levels by analyzing and processing the data $[3,4]$. ADT not only overcomes the disadvantages of the traditional ALT, but also overcomes the disadvantage that ALT only records fault data, so that a reliability evaluation can be carried out under the condition of zero fault [5]. 
Although ADT has a high test efficiency, in order to obtain higher efficiency and reduce test costs, it is usually necessary to optimize the test factors in ADT before test, including sample size, stress level, test time and constraint conditions. In 1962, Chernoff [6] first carried out research on the optimization design of accelerated tests. He studied the optimization design of constant stress accelerated test schemes with an exponential distribution of product life, and gave a theoretical framework for the optimization design of accelerated tests. On the basis of this theoretical framework, Nelson and Meeker [4,7-11] introduced model constraints into the optimization model to improve the theoretical framework of the optimization method. By studying the variation in stress level, sample ratio and asymptotic variance with the model parameters in the experiment, they obtained an optimization scheme which is insensitive to model parameters. However, these studies assume that an object is subjected to a single stress, but a product is often subject to multiple stresses in practice. Therefore, in recent years, scholars have expanded the research content from single stress to multiple stresses. Park [12] applied the idea of single stress optimal design (two stress levels) to an object under the action of two kinds of stresses, researched the optimal design of life distribution obeying the generalized Eyring law, and obtained an optimal scheme based on the factor arrangement of the test points method. Elsayed [13] used the same method to obtain an optimal scheme under the proportional risk model. However, the optimization scheme obtained by this method is highly sensitive to changes in model parameters, and this method only contains two stress levels, which cannot meet the needs of application. Therefore, Hu [14] extended stress levels in SSADT from two to more, and proved the effectiveness of this method under the condition of constraints. Lim [15] studied the validity between model parameters and stress variables, and obtained a method of how to select stress levels in an accelerated test scheme with multiple stress levels. Ge [16] studied the selection of stress levels for multi-stress SSADT under the constraints of test samples and test times. In order to further improve test efficiency, reduce the test sample size and apply to more kinds of products, scholars have expanded the research content from single stress level selection to more test factors. Tsai [17] and Tung [18] researched the ADT optimization method for the degradation process following a gamma process. Mosavebi [19] researched an optimal sample ratio under each sample size and the influence of each stress on the objective function. Sun [20] researched the optimal stress level change time in SSADT, and Li [21] determined the optimal sample size under each stress level, based on different expected utility functions. Wang [22,23] researched the optimization design method of an ADT scheme for multiple degradation processes under multiple stresses using a simulation method. Li [24] researched the optimization design method of ADT when prior information is insufficient, and used the information theory to dynamically modify the test scheme, which effectively shortened the test time. Wang [25] proposed a new optimization criterion, which pays more attention to the equivalence of the degradation mechanism, rather than the evaluation accuracy and prediction accuracy. The optimization results of the criterion were compared with the existing criteria. The results showed that the criterion can effectively reduce the stress distance between the normal stress level and the higher stress level, and caused the test arrangement to be more in line with the practical application. For an object with an uncertain degradation mechanism, Zhao [26] used the competitive failure model to research the optimization method of ADT schemes for products with multiple failure mechanisms, and optimized the established model by using three conventional optimization criteria ( $D$-optimality, a-optimality and v-optimality). The results showed that the optimal scheme under random impact was very different from the traditional ADT optimization scheme. Yu [27] and others have also carried out research on this kind of object. They proposed an improved Bayesian $D$-optimality criterion to solve this kind of problem, and verified the effectiveness of the method through simulations. In order to maximize the efficiency of the ADT test, Jiang [28] optimized the test method to maximize the test stress, and took carbon film resistors as an example to verify the effectiveness of the method. Ma [29] mixed ALT and ADT to reduce test costs to the greatest extent while ensuring accuracy. Wang [30] proposed a 
multi-stress and multi-degradation ADT optimization design method to solve the problem that the existing single degradation measure ADT cannot be applied to expensive and high reliability products. The proposed method, with the evaluation accuracy of the optimization scheme as the optimization objective, along with the stress, stress level, sample size, detection interval and detection time as the optimization variables, the detection time and cost as the constraints, and the product with multiple degradation measures, was studied, and the effectiveness of the method was verified using a rubber sealed O-ring.

However, the above literature regarding the optimization of multi-stress ADT are all about the stress level, sample size, test time and degradation mode. Instead of considering the effect of different stresses on the product, the multiple stresses were directly optimized with the same influence value or influence weight, which leads to the problem of low evaluation accuracy of model parameters of important stresses when using the degradation data generated by the optimized scheme for reliability evaluations. In addition, the main examples of the above ADT scheme optimization methods are electronic components or mechanical products with simple structures, and there are no complex mechanical and electrical products, such as motorized spindles. Therefore, aiming at the problem of how to carry out the multi-stress ADT optimization design of motorized spindles, this paper puts forward an optimization design method of an accelerated test scheme based on $D_{s}$-optimality.

In this paper, based on $D_{s}$-optimality, an optimization method of model parameter accuracy focusing on important stress, is proposed to solve the problem of model parameter evaluation in the ADT of motorized spindles subjected to various stresses. In Section 2, the sensitive stress of a motorized spindle is obtained through SSADT test profile analysis and FTA analysis of the motorized spindle. In Section 3, based on the previous data and the characteristics of motorized spindles, the performance degradation and acceleration model is proposed, and the accelerated degradation model of motorized spindles is established. In Section 4 , based on $D_{S}$-optimality, the optimization objective of this method is established and the solving process is given. In Section 5 , the effectiveness of the method is proved by taking a certain type of motorized spindle made in China as an example, and the results are compared with those of $D$-optimality. In Section 6, the sensitivity of the model parameters in the case is analyzed, and proves the proposed method has good robustness. Section 7 concludes this paper.

\section{SSADT Analysis of Motorized Spindle}

\subsection{SSADT Profile Analysis}

If $I$ kinds of stresses are applied in SSADT of motorized spindle, and each stress has $L$ stress levels, then there are $L^{I}$ kinds of combinations of stress levels in SSADT of motorized spindle. However, according to the characteristics of SSADT [31] $\left(S_{i 1} \leq S_{i 2}, \ldots, \leq S_{i L}\right)$, not all combinations of stress levels can be applied to the test. The SSADT profile is shown in Figure 1. In order to ensure the accuracy of the degradation rate under extrapolated normal stress, it is necessary to adjust the stress level appropriately so that the degradation rate of the motorized spindle increases with the stress loading sequence. Before the test, the degradation rate corresponding to all stress combinations should be calculated so that the degradation rate of the next stress combination in the selected scheme is higher than that of the previous stress combination. In addition, the first stress level $S_{i 1}$ should be close to the normal stress $S_{i 0}$ [32], but the selected $S_{i 1}$ should not be too close to $S_{i 0}$, otherwise it will not be accelerated. The maximum stress level $S_{i l}$ should be as close to the limit level $S_{i \max }$ as possible without changing the failure mechanism, and is generally slightly lower than $S_{i \max }$. If there are $n$ samples in the test, the total test time is $t$, and the test time distribution ratio under the $l$-th stress combination is $r_{l}$, then the test time is $t_{l}=t \times r_{l}(l=1, \ldots, L)$. If the time interval of product performance monitoring is $\Delta t$, the monitoring times are $M_{l}=t_{l} / \Delta t$. 


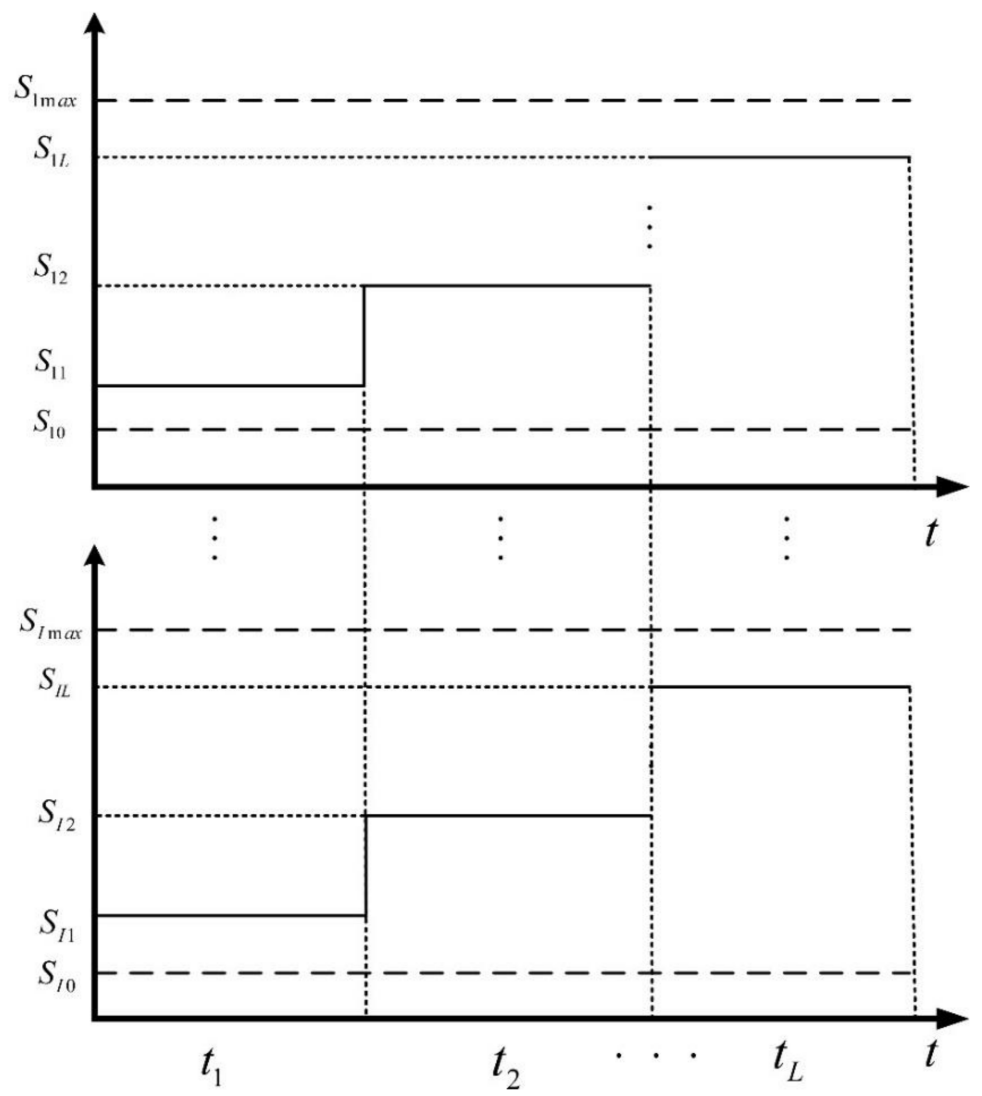

Figure 1. SSADT profile with multiple stresses.

\subsection{SSADT Stress Analysis}

The structure of the motorized spindle is shown in Figure 2. In order to obtain an effective SSADT scheme of the motorized spindle, it is necessary to analyze the stress of the motorized spindle when it works. The data of 520 reliability tests of motorized spindles from a machining center over 3 months (June-August 2016) are summarized, as shown in Table 1.

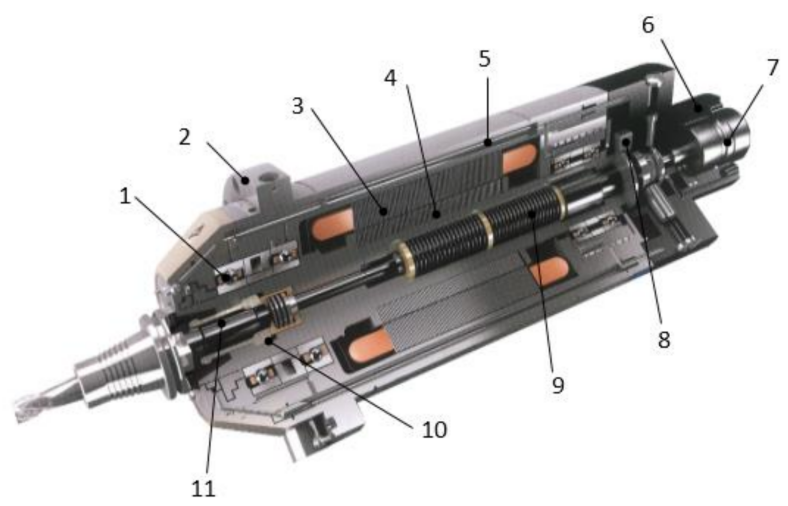

Figure 2. Structure of motorized spindle. 1. Roller bearing; 2. flange; 3. stator; 4. rotor; 5 . cooling line; 6 . lubrication line; 7. hydraulic cylinder; 8. encoder; 9 . disc spring; 10 and 11. broach system. 
Table 1. Failure of motorized spindle system in field reliability test.

\begin{tabular}{|c|c|c|c|c|}
\hline Fault Location & Fault Phenomenon & Fault Cause & $\begin{array}{l}\text { Number of } \\
\text { Times }\end{array}$ & Percentage \\
\hline Bearing & $\begin{array}{l}\text { Spindle swing big, spindle abnormal noise, } \\
\text { workpiece with tool marks, locked, not tight } \\
\text { tool, the cutting tool will not stick }\end{array}$ & $\begin{array}{l}\text { Bearing fatigue and } \\
\text { damage }\end{array}$ & 27 & $67.5 \%$ \\
\hline Positioning block & The cutting tool dropping & Loose and poor preload & 3 & $7.5 \%$ \\
\hline Servo amplifier & sequence error, abnormal bus connection & Servo amplifier failure & 3 & $7.5 \%$ \\
\hline Motor & Large speed deviation, servo system alarm & Motor damage & 2 & $5 \%$ \\
\hline Braking resistance & Brake circuit failure & Brake resistor burned & 2 & $5 \%$ \\
\hline Claw & The spindle cannot hold the cutting tool & Claw damage & 1 & $2.5 \%$ \\
\hline Cone & Abnormal noise of motorized spindle & Cone wear & 1 & $2.5 \%$ \\
\hline Cooling system & Spindle overheat alarm & Water in cooling system & 1 & $2.5 \%$ \\
\hline
\end{tabular}

The fault information of the spindle system in Table 1 is marked in the form of a fault tree, the event label is shown in Table 2, and the fault tree of the established spindle system is shown in Figure 3.

Table 2. Result event label of top event TOP.

\begin{tabular}{|c|c|c|c|}
\hline Event & Code & Event & Code \\
\hline Abnormal noise of motorized spindle & F101 & Wear of inner cone of spindle & E305 \\
\hline The spindle cannot hold the cutting tool normally & F102 & Fault of servo amplifier & E306 \\
\hline Fatigue damage of bearing & E301 & Burn out of brake resistor & E307 \\
\hline Failure of cooling system & E302 & Looseness of positioning block & E308 \\
\hline Abnormal of the motor & E303 & The preload of positioning block is not enough & E309 \\
\hline Claws broken & E304 & & \\
\hline
\end{tabular}

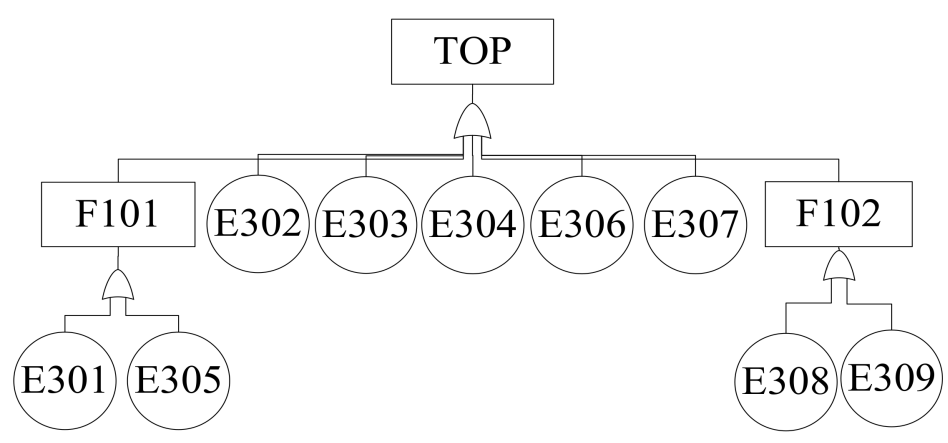

Figure 3. FTA of spindle system.

According to the law of large numbers of Bernoulli [33], if the probability of the bottom event is replaced by the frequency of the bottom event, the frequency of each bottom event in the spindle system is shown in Table 3.

Table 3. Failure probability of each bottom event.

\begin{tabular}{|c|c|c|c|c|c|}
\hline Code & Event & $\begin{array}{l}\text { Probability } \\
\quad\left(* 10^{-2}\right)\end{array}$ & Code & Event & $\begin{array}{c}\text { Probability } \\
\left(* 10^{-2}\right)\end{array}$ \\
\hline E301 & Fatigue damage of bearing & 1.293 & E306 & Fault of servo amplifier & 0.144 \\
\hline E302 & Failure of cooling system & 0.048 & E307 & Burn out of brake resistor & 0.096 \\
\hline E303 & Abnormality of the motor & 0.096 & E308 & Looseness of positioning block & 0.048 \\
\hline E304 & Claws broken & 0.048 & E309 & The preload of positioning block is not enough & 0.096 \\
\hline E305 & Wear of inner cone of spindle & 0.048 & & & \\
\hline
\end{tabular}


The potential improvement potential (IP) method is used to calculate the importance of the bottom event [34], and the calculation formula is shown in Equation (1):

$$
H^{\mathrm{IP}}(R \mid T)=h\left[1_{R}, p(T)\right]-h[p(T)]
$$

where $p_{R}(T)$ is the reliability of bottom event $R(R=1,2, \ldots, N), h[p(T)]$ is the reliability of the system, and $h\left[1_{R}, p(T)\right]$ is the conditional probability of the system working normally when the bottom event $H$ is working normally.

The importance of each event is shown in Table 4.

Table 4. The importance of bottom events.

\begin{tabular}{cccccccccc}
\hline $\begin{array}{c}\text { Bottom } \\
\text { Events }\end{array}$ & E301 & E302 & E303 & E304 & E305 & E306 & E307 & E308 & E309 \\
\hline$H^{I P}(R \mid T)$ & 0.0128 & 0.0005 & 0.0009 & 0.0005 & 0.0005 & 0.0014 & 0.0009 & 0.0005 & 0.0005 \\
\hline
\end{tabular}

As the bottom event $R$ occurs, there is a difference in downtime maintenance time. Therefore, the average downtime $\overline{t_{R}}$ is taken $(R=1,2, \ldots 9)$. The relative downtime coefficient $k_{T}$ is defined:

$$
k_{T}=\frac{\overline{t_{R}}}{\max _{1 \leq R \leq 9}\left(\overline{t_{R}}\right)}
$$

where $\max _{1 \leq R \leq 9}\left(\overline{t_{R}}\right)$ is the longest downtime in the bottom event. In this field reliability test, the average downtime of E304 (claws broken) is the longest, which is $115 \mathrm{~min}$.

Calculate the average downtime $\overline{t_{R}}$ and relative downtime coefficient $k_{T}$ for each bottom event, as shown in Table 5.

Table 5. Average downtime $\overline{t_{R}}$ and relative downtime coefficient $k_{R}$ of each bottom event.

\begin{tabular}{cccccc}
\hline Event & $\overline{\boldsymbol{t}_{\boldsymbol{R}}}(\mathbf{m i n})$ & $k_{T}$ & Event & $\overline{\boldsymbol{t}_{\boldsymbol{R}}}(\mathbf{m i n})$ & $\boldsymbol{k}_{\boldsymbol{T}}$ \\
\hline E301 & 84.41 & 0.6753 & E306 & 83.3 & 0.6664 \\
E302 & 65 & 1 & E307 & 34.5 & 0.276 \\
E303 & 76.5 & 0.612 & E308 & 68.3 & 0.5464 \\
E304 & 115 & 0.92 & E309 & 68.3 & 0.5464 \\
E305 & 80 & 0.64 & & & \\
\hline
\end{tabular}

Because the IP importance considers the influence of the failure rate of the bottom event on the top event, the product of IP importance $H^{\mathrm{IP}}(R \mid T)$ and $k_{T}$ is used to consider the importance of downtime; the calculation formula is:

$$
H^{k_{T}}(R \mid T)=k_{T} \cdot H^{\mathrm{IP}}(R \mid T)
$$

Using Equation (3), the importance of each bottom event considering downtime is calculated as shown in Table 6.

Table 6. Importance of each bottom event considering downtime.

\begin{tabular}{cccc}
\hline Event & $\boldsymbol{H}^{\boldsymbol{k}_{\boldsymbol{T}}}(\boldsymbol{R} \mid \boldsymbol{T})\left(* \mathbf{1 0}^{-\mathbf{2}}\right)$ & Event & $\boldsymbol{H}^{\boldsymbol{k}_{T}}(\boldsymbol{R} \mid \boldsymbol{T})\left(* \mathbf{1 0}^{-\mathbf{2}}\right)$ \\
\hline E301 & 0.9425 & E306 & 0.1021 \\
E302 & 0.0266 & E307 & 0.0282 \\
E303 & 0.0625 & E308 & 0.0279 \\
E304 & 0.0469 & E309 & 0.0558 \\
E305 & 0.0327 & & \\
\hline
\end{tabular}


The cutting force $S_{1}$, spindle speed $S_{2}$, tool change times $S_{3}$, cutting power $S_{4}$, temperature $S_{5}$ and other stresses correspond to the main motorized spindle subsystem, as shown in Figure 4.

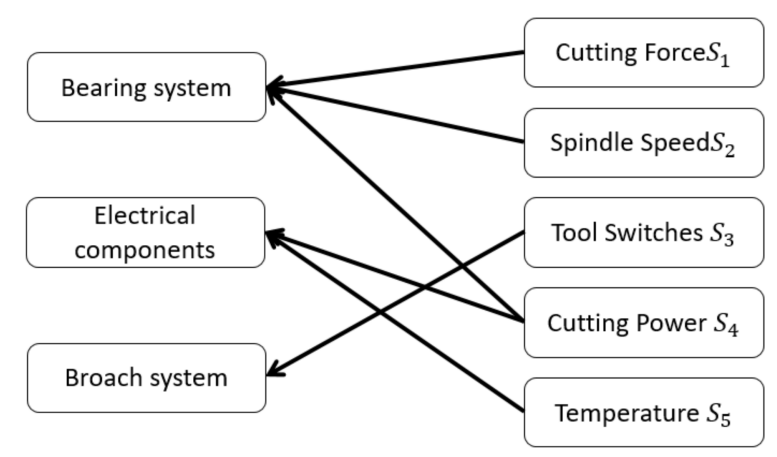

Figure 4. Mapping of stress and spindle subsystem.

According to the mapping relationship in Figure 4 and the failure mechanism of the motorized spindle, the main influence range and effect of stress are obtained, as shown in Table 7.

Table 7. Factors affecting the importance of stress on the motorized spindle.

\begin{tabular}{|c|c|c|c|}
\hline \multirow{2}{*}{ Stress } & \multicolumn{2}{|c|}{ Stress Effect } & \multirow{2}{*}{$H^{a}(R \mid T)\left(* 10^{-2}\right)$} \\
\hline & Main Influence Scope & Stress Action & \\
\hline Cutting force $S_{1}$ & Bearing & 3 & 0.9425 \\
\hline Spindle speed $S_{2}$ & Bearing & 1 & 0.9425 \\
\hline Tool change times $S_{3}$ & Parts of broach system & $\begin{array}{c}1 / w,(w \text { is material constant, } \\
w=9)\end{array}$ & 0.096 \\
\hline Cutting power $S_{4}$ & $\begin{array}{l}\text { All parts in the spindle, among which the } \\
\text { electronic components and bearings are } \\
\text { most affected }\end{array}$ & 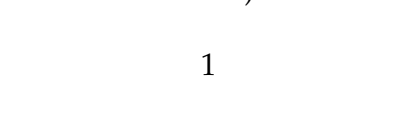 & 0.0133 \\
\hline Temperature $S_{5}$ & $\begin{array}{l}\text { All parts in the spindle, among which the } \\
\text { electronic components are most affected }\end{array}$ & 1 & 0.0133 \\
\hline
\end{tabular}

In Table 7, the main impact range of stress includes five qualitative levels, expressed by $\beta_{Z 0}$, the value of $\beta_{Z 0}$ is marked as an integer from 1 to 5 , and the mark with the widest influence range is 5 . The influence range factor $\beta_{Z}$ of stress is defined as

$$
\beta_{Z}=\frac{\beta_{Z 0}}{\max \left\{\beta_{Z 0}\right\}}
$$

There are three quantitative degrees of stress loading, expressed by $\beta_{0}^{Z}$. the value of $\beta_{0}^{Z}$ ranges from 1 to 3 , marked by integers. Define the action coefficient $\beta^{Z}$ :

$$
\beta^{Z}=\frac{\beta_{0}^{Z}}{\max _{3}\left\{\beta_{0}^{Z}\right\}}
$$

According to Table 7, Equations (4) and (5), the influence range coefficient $\beta_{Z}$ and action coefficient $\beta^{Z}$ are shown in Table 8 . 
Table 8. The influence range coefficient $\beta_{Z}$ and action coefficient $\beta^{Z}$.

\begin{tabular}{ccccc}
\hline Stress & $\beta_{Z 0}$ & $\beta_{Z}$ & $\beta_{0}^{Z}$ & $\beta^{Z}$ \\
\hline Cutting force $S_{1}$ & 1 & 0.2 & 3 & 1 \\
Spindle speed $S_{2}$ & 1 & 0.2 & 1 & $1 / 3$ \\
Tool change times $S_{3}$ & 2 & 0.4 & $1 / 9$ & $1 / 27$ \\
Cutting power $S_{4}$ & 5 & 1 & 1 & $1 / 3$ \\
Temperature $S_{5}$ & 4 & 0.8 & 1 & $1 / 3$ \\
\hline
\end{tabular}

The comprehensive importance of stress $H_{z}(R \mid T)$ is defined:

$$
H_{Z}(R \mid T)=\beta_{Z} \times \beta^{Z} \times H^{\alpha}(R \mid T)
$$

According to Equation (6), the comprehensive importance of the stress of the motorized spindle is obtained, as shown in Table 9.

Table 9. Comprehensive importance of stress.

\begin{tabular}{cc}
\hline Stress & $\boldsymbol{H}_{\mathbf{Z}}(\boldsymbol{R} \mid \mathbf{T}) \mathbf{1 \mathbf { 1 0 } ^ { - \mathbf { 2 } }}$ \\
\hline Cutting force $S_{1}$ & 0.189 \\
Spindle speed $S_{2}$ & 0.063 \\
Tool change times $S_{3}$ & 0.001 \\
Cutting power $S_{4}$ & 0.004 \\
Temperature $S_{5}$ & 0.003 \\
\hline
\end{tabular}

According to Table 9, it can be concluded that the order of importance of each stress on the reliability of motorized spindle is as follows: cutting force $>$ spindle speed $>$ cutting power $>$ temperature $>$ tool change times.

\section{Performance Degradation and Acceleration Model of Motorized Spindle}

\subsection{Degradation Model}

Drift Brownian motion is used to model the degradation process of the motorized spindle. The generalized path model is used to describe the degradation process of the motorized spindle [11]:

$$
y_{i, l, j}\left(t_{k}\right)=\sigma_{1} B\left(t_{k}\right)+\mu_{k} t_{k}+y_{0}
$$

where $y_{i, l, j}\left(t_{k}\right)$ is the performance degradation value of the $j$-th sample at the stress $S_{i}$ level $l$ at time $t_{k}\left(l=1,2, \ldots, L, L\right.$ is the number of stress levels, $j=1,2, \ldots, n_{l}, n_{l}$ is the sample size under the stress level, $k=1,2, \ldots, m_{l}, m_{l}$ is the number of sample measurements at the stress $S_{i}$ level $\left.l\right), B\left(t_{k}\right)$ is the standard Brownian motion, $B\left(t_{k}\right) \sim N\left(0, t_{k}\right), \sigma_{1}$ is the diffusion coefficient, which is used to describe the differences in samples, operation and environmental conditions of the motorized spindle test; therefore, $\sigma_{1}$ does not change with the change of stress and time. $\mu_{k}$ is the drift coefficient, also known as performance degradation rate. $y_{0}$ is the starting point of drift Brownian motion, which is the initial value of sample performance.

\subsection{Acceleration Model}

According to the results in Section 2.2, it can be concluded that the stress of the motorized spindle is mainly mechanical stress and electrical stress, which is close to the stress described by the inverse power law model [35]. Therefore, the inverse power law model is selected as the acceleration model of the motorized spindle acceleration test, and its standard equation is as follows:

$$
P=C S_{l}^{A}
$$

where $P$ is the reliability characteristic quantity, $S_{l}$ is the $l$-th stress level of the stress, and C and $\mathrm{A}$ are constants independent of the stress level, but related to the failure model and stress type. 
The standard inverse power law model can only be used as an acceleration model for a single stress. In order to make it applicable to the case of multiple stresses, the standard inverse power law model is extended [35]:

$$
P=C S_{1 l}^{A 1} S_{2 l}^{A 2} \ldots S_{I l}^{A I}
$$

Take a logarithm on both sides of Equation (9), and Equation (9) becomes:

$$
\ln P=A 1 \ln S_{1 l}+A 2 \ln S_{2 l}+\ldots+A I \ln S_{I l}+\ln C
$$

The performance degradation or failure of motorized spindle is usually related to a variety of stresses. In Reference [4], a generalized logarithmic linear model was established by means of stress combination, as shown in Equation (11):

$$
\ln (\mu)=\gamma_{1} \varphi_{1}+\ldots+\gamma_{I} \varphi_{I}+\gamma_{0}
$$

where $\gamma_{0}, \gamma_{1}, \ldots, \gamma_{s}$ are the model parameter and $\varphi_{1}, \varphi_{2}, \ldots \varphi_{n}$ are the stress function.

Since $P$ is the reliability characteristic, then in accelerated degradation test, $P=\mu$, if $\ln C=\gamma_{0}, A 1=\gamma_{1}, \ldots, A I=\gamma_{I}$, by combining Equation (10) with Equation (11), a generalized logarithmic acceleration model between the motorized spindle and stress is obtained, as shown in Equation (12):

$$
\ln (\mu)=\gamma_{0}+\gamma_{1} \ln S_{1 l}+\ldots+\gamma_{I} \ln S_{I l}+\gamma_{0}
$$

Based on the above analysis and assumption, the accelerated degradation model of the spindle is established, as shown in Equation (13):

$$
\left\{\begin{array}{c}
\ln \mu_{k}=\gamma_{1} \ln S_{1 l}+, \ldots,+\gamma_{I} \ln S_{I l}+\gamma_{0} \\
Y \sim N\left(\mu_{k}\left(\Delta t_{k}\right), \sigma^{2}\left(\Delta t_{k}\right)\right)
\end{array}\right.
$$

where $\Delta t_{k}=t_{k}-t_{k-1}$

\section{Optimal Design}

\subsection{Optimization Objective}

It is assumed that the motorized spindle has $L$ stress levels under stress $S_{i}(i=1, \ldots, I)$, the $k$-th performance test time of the $j$ sample under group $l$ stress is $t_{i l j k}(l=1,2, \ldots, L$, $L$ is the number of stress levels; $j=1,2, \ldots, n_{l}, n_{l}$ is the sample size under the stress level $l ; k=1,2, \ldots, m_{l}, m_{l}$ is the inspection times under the stress level $l$ ), the detected performance value is $y_{l j k}$.

Brownian motion is a Gaussian process. Therefore, the performance increment $\Delta y=y_{l j k}-y_{l j(k-1)}$ is independent and follows normal distribution. According to Equation (13), the probability density function of independent increment is as follows:

$$
f(x)=\frac{1}{\sigma \sqrt{2 \pi \Delta t}} \exp \left\{-\frac{[x-\mu \times \Delta t]^{2}}{2 \sigma^{2} \times \Delta t}\right\}
$$

The logarithmic likelihood function of Equation (14) is shown in Equation (15):

$$
\ln p(x \mid \theta) \propto-\frac{1}{2} \sum_{l=1}^{L} \sum_{j=1}^{n_{l}} \sum_{k=1}^{m_{l}}\left\{\left[\ln (2 \pi \Delta t)+\ln \left(\sigma^{2}\right)\right]+\frac{\left[x_{l j k}-\mu_{k} \times \Delta t\right]^{2}}{\sigma^{2} \Delta t}\right\}
$$

where $\theta=\gamma_{0}, \gamma_{1}, \ldots, \gamma_{I}$. For SSADT of the motorized spindle, the sample size under each stress combination is the same, $n_{l}=(1 / l) \times n$ ( $n$ is the total number of samples).

$D_{s}$-optimality is the generalization of $D$-optimality, which is an information-based optimization method. For the model with more parameters, it focuses on the evaluation 
accuracy of important parameters. In other words, only some important parameters in $\theta$ are estimated. Therefore, Equation (6) is divided into two parts:

$$
\ln (\mu)=g_{1}(x)^{\prime} \theta_{1}+g_{2}(x)^{\prime} \theta_{2}
$$

where $\theta_{1}$ contains $s$ important parameters $\left(\theta_{1}=\gamma_{1}, \ldots, \gamma_{s}\right), \theta_{2}$ contains $(L-s)$ unimportant parameters $\left(\theta_{2}=\gamma_{s+1}, \ldots, \gamma_{I}, \gamma_{0}\right)$. Therefore, $g_{1}(x)^{\prime}=\left(\ln S_{1 l}, \ldots, \ln S_{s l}\right), g_{2}(x)^{\prime}=$ $\left(\ln S_{s+1, l}, \ldots, \ln S_{I L}, 1\right)$.

Based on $D_{\mathcal{S}}$-optimality, the information matrix $M_{\tilde{\xi}}\left(M_{\tilde{\xi}}=\operatorname{det}[F(\theta)]\right)$ is divided into four parts

$$
M_{\xi}=\left[\begin{array}{ll}
M_{11}(\xi) & M_{12}(\xi) \\
M_{21}(\xi) & M_{22}(\xi)
\end{array}\right]
$$

where $\xi$ is the selected test scheme.

According to the concept of the information matrix [36], we can get the following conclusions:

$$
M_{\tilde{\zeta}}=G^{\prime} G / L
$$

where $G=\left[\begin{array}{ccc}\varphi_{11} & \ldots & \varphi_{1 \mathrm{~L}} \\ \vdots & & \vdots \\ \varphi_{\mathrm{I} 1} & \cdots & \varphi_{\mathrm{IL}}\end{array}\right]$.

Therefore:

$$
\mathrm{M}_{\xi}=\left[\begin{array}{cccc}
\mathrm{E}\left(-\frac{\partial^{2} \ln p(x \mid \theta)}{\partial^{2} \gamma_{1}}\right) & \ldots & \mathrm{E}\left(-\frac{\partial^{2} \ln p(x \mid \theta)}{\partial \gamma_{1} \partial \gamma_{0}}\right) & \mathrm{E}\left(-\frac{\partial^{2} \ln p(x \mid \theta)}{\partial \gamma_{1} \partial^{2}}\right) \\
\mathrm{E}\left(-\frac{\partial^{2} \ln p(x \mid \theta)}{\partial \gamma_{2} \partial \gamma_{1}}\right) & & \mathrm{E}\left(-\frac{\partial^{2} \ln p(x \mid \theta)}{\partial \gamma_{2} \partial \gamma_{0}}\right) & \mathrm{E}\left(-\frac{\partial^{2} \ln p(x \mid \theta)}{\partial \gamma_{2} \partial \sigma^{2}}\right) \\
\vdots & \ddots & & \vdots \\
\mathrm{E}\left(-\frac{\partial^{2} \ln p(x \mid \theta)}{\partial \gamma_{0} \partial \gamma_{1}}\right) & \vdots & \mathrm{E}\left(-\frac{\partial^{2} \ln p(x \mid \theta)}{\partial^{2} \gamma_{0}}\right) & \mathrm{E}\left(-\frac{\partial^{2} \ln p(x \mid \theta)}{\partial \gamma_{0} \partial \sigma^{2}}\right) \\
\mathrm{E}\left(-\frac{\partial^{2} \ln p(x \mid \theta)}{\partial \sigma^{2} \partial \gamma_{1}}\right) & & \mathrm{E}\left(-\frac{\partial^{2} \ln p(x \mid \theta)}{\partial \sigma^{2} \partial \gamma_{0}}\right) & \mathrm{E}\left(-\frac{\partial^{2} \ln p(x \mid \theta)}{\left(\partial \sigma^{2}\right)^{2}}\right)
\end{array}\right]
$$

The covariance matrix of parameter $\theta_{1}$ is the $s \times s$ sub matrix of the upper left part of matrix $M^{-1}(\xi)$, denoted as $M^{11}(\xi)$, according to the inverse matrix of block matrix, Equation (20) becomes:

$$
M^{11}(\xi)=\left[M_{11}(\xi)-M_{12}(\xi) M_{22}^{-1}(\xi) M_{21}(\xi)^{\prime}\right]^{-1}
$$

Then, the $D_{s}$-optimal design of $\theta_{1}$ is the minimization of the determinant of Equation (20), i.e.:

$$
\max \left|M_{11}(\xi)-M_{12}(\xi) M_{22}^{-1}(\xi) M_{21}(\xi)^{\prime}\right|=\max \frac{|M(\xi)|}{\left|M_{22}(\xi)\right|}
$$

\subsection{Optimization Variables and Constraint Conditions}

\subsubsection{Optimization Variables}

Each element of SSADT can be taken as one of the optimization variables as follow:

- Stress levels $S_{i l}$ and their combinations $\left[\left(S_{11}, S_{21}, \ldots, S_{I 1}\right), \ldots,\left(S_{I 1}, S_{I 2}, \ldots, S_{I L}\right)\right]$;

- $\quad$ Sample size $n_{l}$ under the stress level $l$;

- Inspection times $m_{l}$ under the stress level $l$.

\subsubsection{Constraint Conditions}

The constraint conditions can be divided into test cost constraint and test variable actual value range constraint, in which the test cost can be divided into sample cost and test cost. The sample cost is the product of sample unit price and sample number. The 
test cost includes the depreciation of test box and test equipment, and the consumption of human and power resources, that is, the product of test unit price and test time.

Therefore, the constraint expression of test cost is as follows:

$$
C_{1} \geq n \cdot C_{d}+C_{0} \cdot \sum_{l=1}^{L} m_{l} \times \Delta t
$$

where $C_{1}$ is the total cost of the test and $C_{d}$ is the unit price of the test motorized spindle. $C_{0}$ is the cost of the test per unit time (including inspection costs).

The constraints of the actual value range of test variables mainly include stress level, inspection times and the number of samples under each stress level. Among them:

1. $S_{i 0}<S_{i 1}<S_{i 2}<S_{i 3} \cdots<S_{i L}<S_{i \max }, S_{i 0}$ is the normal stress when the motorized spindle is working, and $S_{i \max }$ is the ultimate stress when the motorized spindle is working. The ultimate stress can be obtained through the technical requirements of the motorized spindle, and then the highest stress $S_{i L}$ is selected according to the ultimate stress so as to ensure that the failure mechanism of the motorized spindle remains unchanged at all stress levels;

2. Inspection times $m_{1} \geq m_{2} \geq \cdots \geq m_{l} \geq 10, m_{1} \geq m / l, \sum_{l=1}^{L} m_{l}=m$, the degradation of motorized spindle is a slow process. In order to ensure the regression fitting accuracy of degradation, the inspection times under each stress level should not be less than 10; under the premise of unchanged failure mechanism, the degradation rate of motorized spindle will accelerate with the increase of stress. In order to collect enough degradation information under each stress level, the inspection times under low stress level should be more than high Stress level.

3. $n_{l} \geq 5$, in order to ensure the statistical significance of the data obtained in the test, the number of test samples under each stress should not be less than 5 .

By summarizing the above constraints, the mathematical expression of the constraint conditions of the test scheme can be expressed as follows:

$$
\text { s.t. }\left\{\begin{array}{c}
C_{1} \geq n \cdot C_{d}+C_{0} \cdot \sum_{l=1}^{L} m_{l} \\
S_{i 0}<S_{i 1}<S_{i 2}<S_{i 3} \cdots<S_{i L}<S_{i m a x} \\
m_{1} \geq m_{2} \geq \cdots \geq m_{l} \geq 10, m_{1} \geq \frac{m}{l} \\
n_{l} \geq 5
\end{array}\right.
$$

Thus, the optimization problem can be described as:

$$
\begin{gathered}
\max \frac{|M(\xi)|}{\left|M_{22}(\xi)\right|} \\
C_{1} \geq n \cdot C_{d}+C_{0} \cdot \sum_{l=1}^{L} m_{l} \cdot \Delta t \\
\text { s.t. } \quad\left\{\begin{array}{c}
S_{i 0}<S_{i 1}<S_{i 2}<S_{i 3} \cdots<S_{i L}<S_{i m a x} \\
m_{1} \geq m_{2} \geq \cdots \geq m_{l} \geq 10, m_{1} \geq \frac{m}{l} \\
n_{l} \geq 5
\end{array} \quad \text { variables }: S_{i l}, n_{l}, m_{l}\right.
\end{gathered}
$$

\subsection{Optimal Process}

In order to avoid the wrong solution caused by improper setting of initial conditions, this paper uses exhaustive method to solve all the test schemes. The specific optimization flow chart is shown in Figure 5. The specific steps are as follows:

1. According to the historical data, engineering experience and other information, the parameters of the model, the degradation threshold $D$ of the motorized spindle performance [37], the initial stress $S_{1}$ and the maximum stress $S_{L}$ of the motorized spindle test, the number of test stress levels $L$, the total test cost $C_{1}$, the test cost per unit time $C_{0}$, the unit price of motorized spindle sample $C_{d}$ and the inspection interval $\Delta t$ are determined; 
2. The test stress level combination $S_{i l}$, sample ratio $n_{l}$ and monitoring times $m_{l}$ under each stress were solved which satisfied the constraints, then the results of them constitute test scheme set $\Omega_{\tilde{\zeta}} . \xi_{q}=\left\{\left[n_{1}, n_{2}, \ldots, n_{l}\right] ;\left[S_{11}, S_{12}, \ldots, S_{i(l-1)}, S_{i l}\right] ;\left[m_{1}, m_{2}, \ldots, m_{l}\right]\right\}$, $q=1,2, \ldots, N_{\xi}, N_{\xi}$ is the total number of schemes in the scheme set;

3. Select a scheme $\xi_{q}$ from scheme set $\Omega_{\xi}$;

4. According to Equation (13), the performance degradation data $Y_{q w}$ of motorized spindle is simulated using the Monte Carlo method for $N_{m}$ times;

5. The information matrix $M(\xi)$ of degradation data $Y_{q w}$ of each simulation is calculated;

6. According to Equation (20), the objective function value $\left|M^{11}(\xi)\right|$ of the scheme $\xi_{q}$ is calculated;

7. Repeat steps 3-6 to calculate the objective function for all schemes in the scheme set;

8. The scheme $\xi^{*}$ with the minimum objective function $\left|M^{11}(\xi)\right|$ is selected as the optimal scheme.

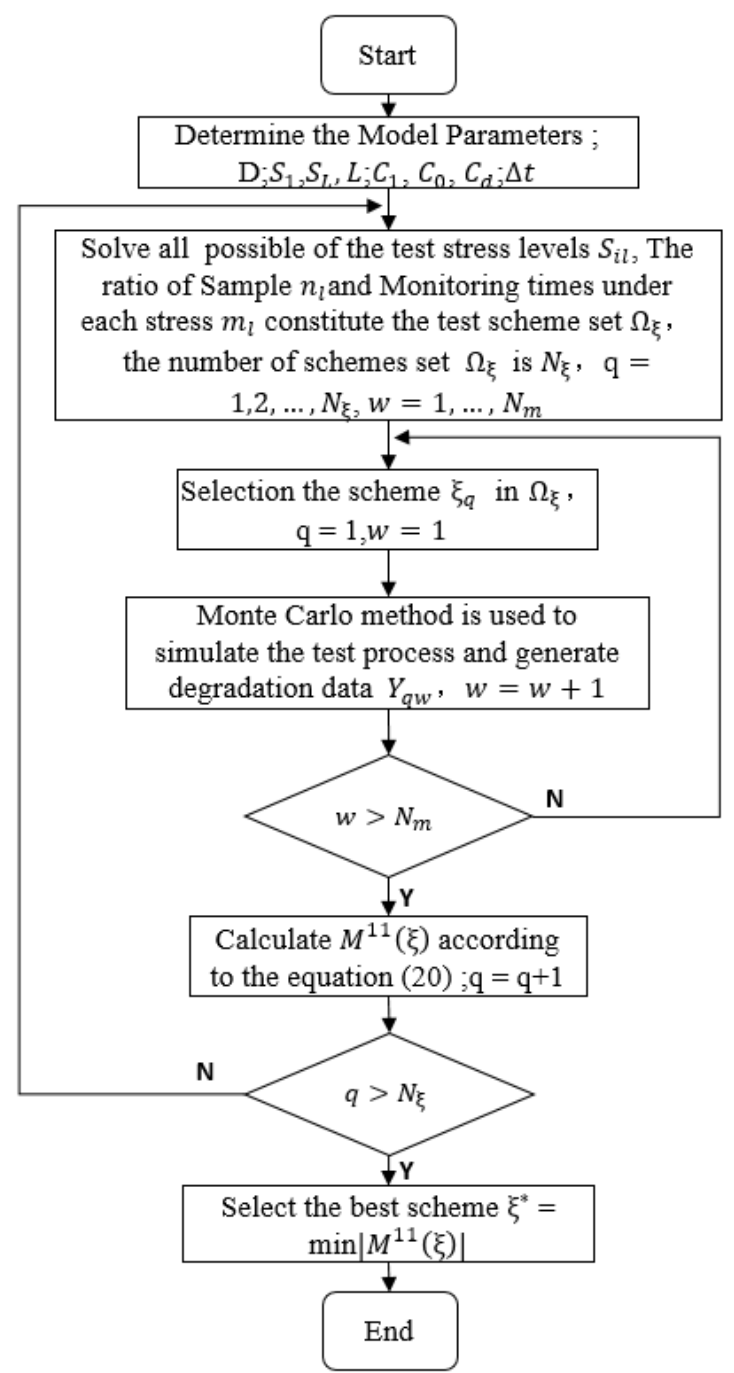

Figure 5. Optimization process of accelerated degradation test scheme for the motorized spindle.

\section{Case Study}

\subsection{A Case Study of Motorized Spindle}

Taking the motorized spindle produced by Sipu Company in China as an example, the proposed optimization method was applied. The parameters of the spindle are shown in Table 10. The parameters of the accelerated degradation model of the motorized spindle obtained from previous reliability test (December 2016-December 2019) are shown in Table 11. 
Table 10. Technical parameters of the sample motorized spindle.

\begin{tabular}{ccccccc}
\hline Type & $\begin{array}{c}\text { Rated Power } \\
\boldsymbol{P}_{\boldsymbol{e}} / \mathbf{k W}\end{array}$ & $\begin{array}{c}\text { Rated Voltage } \\
\boldsymbol{U}_{\boldsymbol{e}} / \boldsymbol{V}\end{array}$ & $\begin{array}{c}\text { Rated Current } \\
\boldsymbol{I}_{\boldsymbol{e}} / \boldsymbol{A}\end{array}$ & $\begin{array}{c}\text { Rated Torque } \\
\boldsymbol{T}_{\boldsymbol{e}} / \mathrm{Nm}\end{array}$ & $\begin{array}{c}\text { Rated Speed } \\
\boldsymbol{n}_{\boldsymbol{e}} / \mathrm{r} \boldsymbol{m} \boldsymbol{m}\end{array}$ & $\begin{array}{c}\text { Frequency } \\
\boldsymbol{f}_{\boldsymbol{m}} / \mathrm{Hz}\end{array}$ \\
\hline SPS1530-18Z & 7.3 & 350 & 15 & 5.9 & 12000 & 300 \\
\hline
\end{tabular}

Table 11. Parameters of accelerated degradation model of the motorized spindle under double stress.

\begin{tabular}{cccc}
\hline$\gamma_{1}$ & $\gamma_{2}$ & $\gamma_{0}$ & $\sigma$ \\
\hline 0.0875 & 0.3423 & -11.6428 & 0.006339 \\
\hline
\end{tabular}

By substituting the model parameters in Table 11 into Equation (13):

$$
\left\{\begin{aligned}
& \ln \mu_{k}= 0.0875 \times \ln S_{1 l}+0.3423 \times \ln S_{2 l}-11.6428 \\
& Y \sim N\left(\mu_{k}\left(\Delta t_{k}\right),(0.006339)^{2}\left(\Delta t_{k}\right)\right)
\end{aligned}\right.
$$

As the motorized spindle is a complex electromechanical system, in order to adapt to its complex failure mechanism, the linear Brownian motion of Equation (25) is transformed into nonlinear Brownian motion, and the $\Delta t_{k}$ in Equation (25) is transformed into $\Delta t_{k}{ }^{\lambda}$ [38], where $\lambda=1.506$.

According to Section 2.2, the cutting force $S_{1}$ and rotation speed $S_{2}$ are selected as the test stress. According to the technical parameter information, subsystem fault characterization and engineering experience, the failure threshold of motorized spindle is determined as $30 \mu \mathrm{m}$ The maximum cutting stress $S_{1 L}$ is $1000 \mathrm{~N}$, and the maximum spindle speed stress $S_{2 L}$ is 10,000 r pm; in order to ensure the acceleration of the test, the minimum cutting stress $S_{11}$ is set to $500 \mathrm{~N}$ and the minimum spindle speed stress $S_{22}$ is set to $5000 \mathrm{rpm}$ according to the previous working conditions. The surface fitting diagram of accelerated degradation model is shown in Figure 6. The coordinate point $X$ is the value of speed $S_{2}, \mathrm{Y}$ is the value of cutting force $S_{1}$, and $\mathrm{Z}$ is the value of $\mu_{k}$. Figure 7 shows the projection of equation surface fitting Figure 6 on the plane, that is, the stress contour line. According to Figure 7, it can be seen that the influence of cutting force on degradation rate is far greater than that of rotation speed, which is consistent with the conclusion of stress analysis in Section 2.2.

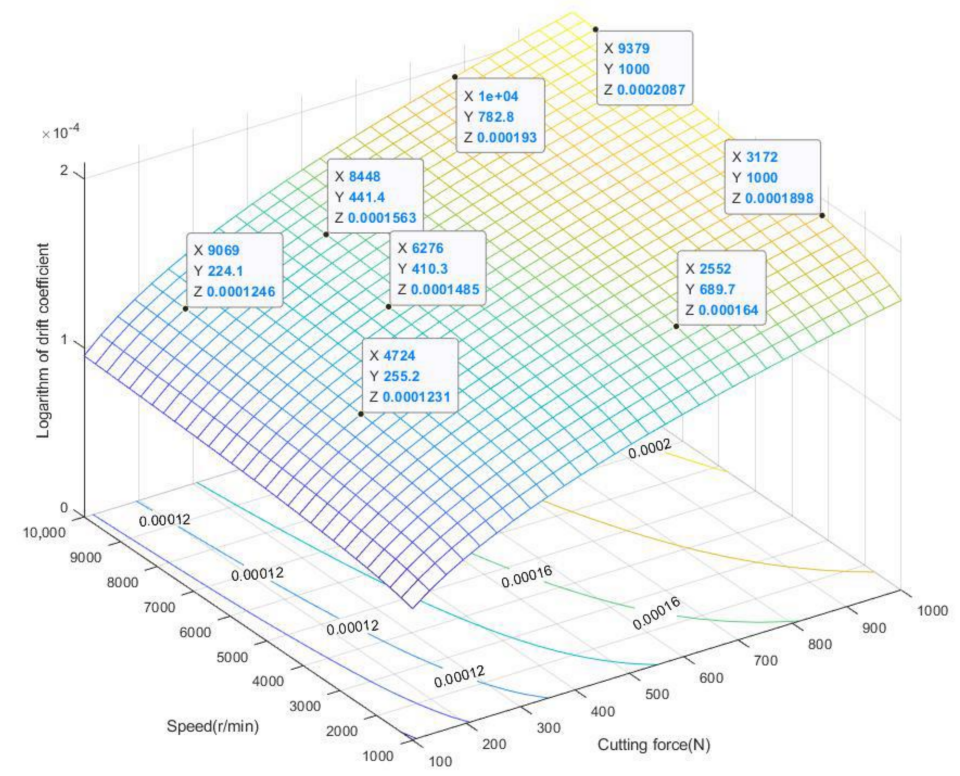

Figure 6. Three-dimensional fitting of the accelerated degradation model of the motorized spindle under two stresses. 


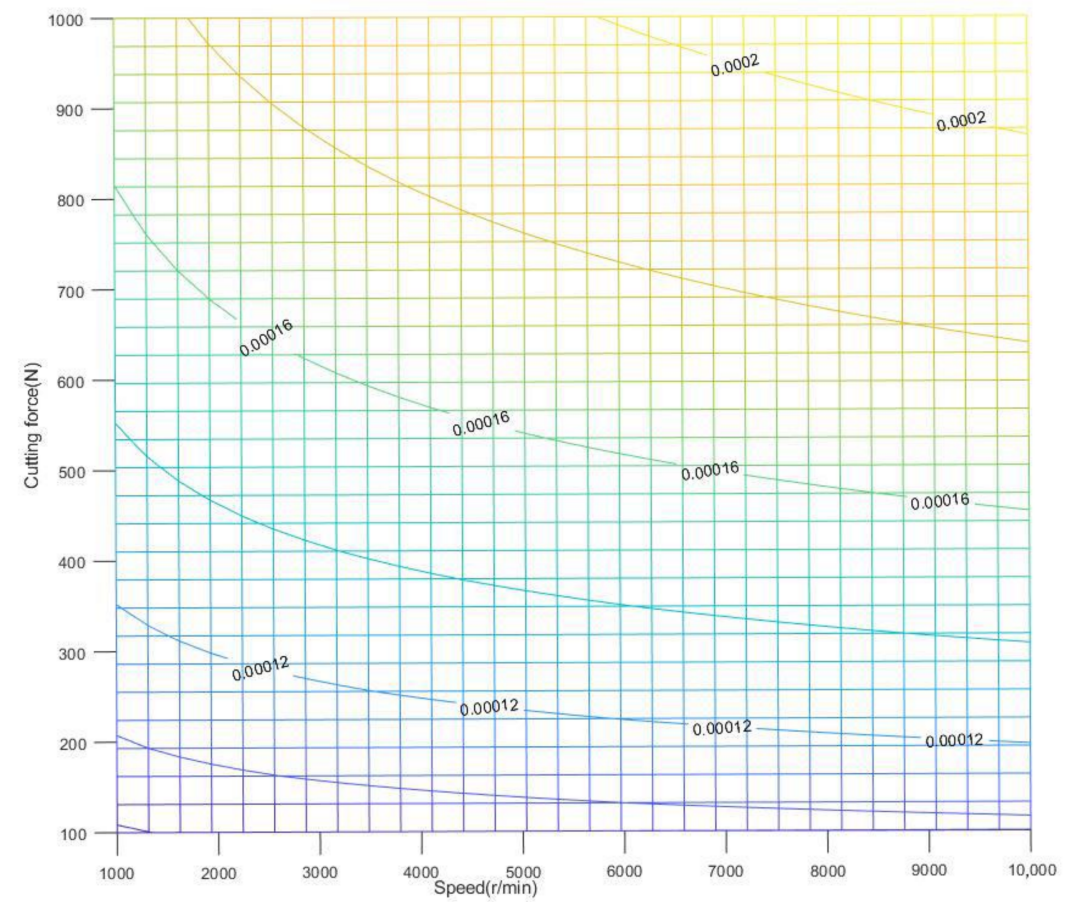

Figure 7. Stress contour of the model.

According to the $D_{S}$-optimality and the actual situation of the calculation example, the cutting force is selected as the main stress concerned $\left(M_{m}^{11}(\xi)\right.$ is $1 \times 1$ matrix), and the information matrix $M_{\xi}$ is

$$
M_{\mathcal{\zeta}}^{m}=\left[\begin{array}{llll}
E\left(-\frac{\partial^{2} \ln p(x \mid \theta)}{\partial^{2} \gamma_{1}}\right) & E\left(-\frac{\partial^{2} \ln p(x \mid \theta)}{\partial \gamma_{1} \partial \gamma_{2}}\right) & E\left(-\frac{\partial^{2} \ln p(x \mid \theta)}{\partial \gamma_{1} \partial \gamma_{0}}\right) & E\left(-\frac{\partial^{2} \ln p(x \mid \theta)}{\partial \gamma_{1} \partial \sigma^{2}}\right) \\
E\left(-\frac{\partial^{2} \ln p(x \mid \theta)}{\partial \gamma_{2} \partial \gamma_{1}}\right) & E\left(-\frac{\partial^{2} \ln p(x \mid \theta)}{\partial^{2} \gamma_{2}}\right) & E\left(-\frac{\partial^{2} \ln p(x \mid \theta)}{\partial \gamma_{2} \partial \gamma_{0}}\right) & E\left(-\frac{\partial^{2} \ln p(x \mid \theta)}{\partial \gamma_{2} \partial \sigma^{2}}\right) \\
E\left(-\frac{\partial^{2} \ln p(x \mid \theta)}{\partial \gamma_{0} \partial \gamma_{1}}\right) & E\left(-\frac{\partial^{2} \ln p(x \mid \theta)}{\partial \gamma_{0} \partial \gamma_{2}}\right) & E\left(-\frac{\partial^{2} \ln p(x \mid \theta)}{\partial^{2} \gamma_{0}}\right) & E\left(-\frac{\partial^{2} \ln p(x \mid \theta)}{\partial \gamma_{0} \partial \sigma^{2}}\right) \\
E\left(-\frac{\partial^{2} \ln p(x \mid \theta)}{\partial \sigma^{2} \partial \gamma_{1}}\right) & E\left(-\frac{\partial^{2} \ln p(x \mid \theta)}{\partial \sigma^{2} \partial \gamma_{2}}\right) & E\left(-\frac{\partial^{2} \ln p(x \mid \theta)}{\partial \sigma^{2} \partial \gamma_{0}}\right) & E\left(-\frac{\partial^{2} \ln p(x \mid \theta)}{\partial\left(\sigma^{2}\right)^{2}}\right)
\end{array}\right]
$$

Because $M_{m}^{11}(\xi)$ is $1 \times 1$ matrix, and according to the principle of matrix partition, $M_{22}^{m}(\xi)$ can be divided into

$$
M_{22}^{m}(\xi)=\left[\begin{array}{lll}
E\left(-\frac{\partial^{2} \ln p(x \mid \theta)}{\partial^{2} \gamma_{2}}\right) & E\left(-\frac{\partial^{2} \ln p(x \mid \theta)}{\partial \gamma_{2} \partial \gamma_{0}}\right) & E\left(-\frac{\partial^{2} \ln p(x \mid \theta)}{\partial \gamma_{2} \partial \sigma^{2}}\right) \\
E\left(-\frac{\partial^{2} \ln p(x \mid \theta)}{\partial \gamma_{0} \partial \gamma_{2}}\right) & E\left(-\frac{\partial^{2} \ln p(x \mid \theta)}{\partial^{2} \gamma_{0}}\right) & E\left(-\frac{\partial^{2} \ln p(x \mid \theta)}{\partial \gamma_{0} \partial \sigma^{2}}\right) \\
E\left(-\frac{\partial^{2} \ln p(x \mid \theta)}{\partial \sigma^{2} \partial \gamma_{2}}\right) & E\left(-\frac{\partial^{2} \ln p(x \mid \theta)}{\partial \sigma^{2} \partial \gamma_{0}}\right) & E\left(-\frac{\partial^{2} \ln p(x \mid \theta)}{\partial\left(\sigma^{2}\right)^{2}}\right)
\end{array}\right]
$$

The objective function of scheme $\xi$ is obtained as follows

$$
M_{m}^{11}(\xi)=\frac{M_{\xi}^{m}}{M_{22}^{m}(\xi)}
$$

According to engineering experience and test experience, the cost of accelerated degradation test for motorized spindle is shown in Table 12. 
Table 12. Expected test cost and accelerated degradation test cost.

\begin{tabular}{cc}
\hline Item & Cost (Ten Thousand Yuan) \\
\hline Expected cos t of testing $\left(C_{1}\right)$ & 75 \\
$C_{d}$ & 2.5 \\
$C_{0}$ & $0.01 / \mathrm{h}$ \\
\hline
\end{tabular}

According to Reference [39], for SSADT with two stresses, when the number of stress levels is 4 , the evaluation of the test scheme is the highest. Therefore, in order to ensure the accuracy and effectiveness of extrapolation, four stress levels (i.e., $L=4$ ). According to the objective function, Equation (28), all possible scheme sets are solved, and the optimal test scheme under each sample size $(n=20, n=24, n=28)$ is obtained, as shown in Table 13.

Table 13. Optimization results of test scheme.

\begin{tabular}{cccccc}
\hline Number & $\boldsymbol{n}$ & $\boldsymbol{S}_{1}$ & $\mathbf{m}$ & & $|\boldsymbol{M}(\boldsymbol{\xi})| /\left|\boldsymbol{M}_{22}(\boldsymbol{\xi})\right|$ \\
\hline 1 & {$[5555]$} & {$[800850900950]$} & {$[6000650070007500]$} & {$[66626161]$} & 1651636.372 \\
2 & {$[66666]$} & {$[800850900950]$} & {$[6000650070007500]$} & {$[40383636]$} & 455879.2074 \\
3 & {$[7777]$} & {$[800850900950]$} & {$[6000650070007500]$} & {$[16121111]$} & 31610.36 \\
\hline
\end{tabular}

From the results in Table 13 that under the same constraint conditions, the scheme with the largest objective function of the motorized spindle accelerated degradation test with different sample sizes ( $n=20, n=24, n=28)$ has the same stress level, i.e., cutting force $S_{1}=$ [800 850900 950], speed $S_{2}=[6000650070007500]$. However, due to the constraint of test cost, the inspection times are very different (i.e., the test time is very different), and then the objective function is very different. Therefore, under the same cost constraint condition, the test time should be extended as much as possible, instead of pursuing large samples, when the $D_{s}$-optimality is used to optimize the multi stress accelerated degradation test of motorized spindle.

In order to check the evaluation accuracy of other model parameters, the objective function of the test scheme with the largest objective function (Number 1 in Table 13) is calculated under the traditional model parameter optimization criterion $D$-optimality, and the optimization based on $D$-optimality is calculated under the same conditions. The results are shown in Table 14.

Table 14. D-optimality optimal scheme.

\begin{tabular}{|c|c|c|c|c|c|}
\hline Number & $n$ & $S_{1}$ & $S_{2}$ & m & $\operatorname{det}|M(\mathcal{\xi})|$ \\
\hline 1 & {$\left[\begin{array}{llll}5 & 5 & 5 & 5\end{array}\right]$} & [800 850900 950] & [6000 650070007500$]$ & {$\left[\begin{array}{lllll}6 & 62 & 61 & 61\end{array}\right]$} & $4.29 * 10^{24}$ \\
\hline 2 & {$\left[\begin{array}{llll}5 & 5 & 5 & 5\end{array}\right]$} & {$\left[\begin{array}{lllll}600 & 800 & 850 & 900\end{array}\right]$} & [5000 550060007000$]$ & {$\left[\begin{array}{llll}117 & 113 & 10 & 10\end{array}\right]$} & $5.26 * 10^{24}$ \\
\hline
\end{tabular}

It can be seen from Table 14 that the optimal scheme obtained by $D_{s}$-optimality optimization can also obtain higher objective function value under $D$-optimality, which means that the multi stress accelerated degradation test scheme of motorized spindle obtained by multi stress optimization based on $D$-optimality can also obtain higher estimation accuracy for other model parameters. Therefore, this optimization method can not only maintain the accuracy of other stress parameters evaluation, but also improve the evaluation accuracy of main stress model parameters, so as to solve the problem that the evaluation of important parameters is not accurate due to too many model parameters in multi stress accelerated degradation test scheme of motorized spindle.

\subsection{An Extended Case Study}

In order to ensure that the method can be applied to the SSADT scheme optimization design of other products under multi stress, the case study in Reference [39] is taken as 
a case study to carry out the optimization design. The accelerated degradation model of products in Reference [39] is as follows:

$$
\left\{\begin{array}{c}
\ln \mu_{k}^{1}=A^{1} / T+B^{1} * \ln V+C^{1} \\
Y
\end{array}\right.
$$

In Equation (29), $T$ is absolute temperature, in $\mathrm{K} ; V$ is voltage, in $\mathrm{V} ; A^{1}, B^{1}, C^{1}$ are the model parameters; the specific values of $A^{1}, B^{1}, C^{1}$ and $\sigma_{1}$ are shown in Table 15 .

Table 15. Specific values of model parameters.

\begin{tabular}{cccc}
\hline$A^{1}$ & $\boldsymbol{B}^{1}$ & $C^{1}$ & $\sigma_{1}$ \\
\hline-8704 & 0.5 & 10 & 0.002 \\
\hline
\end{tabular}

Substitute the parameter values into Equation (29):

$$
\left\{\begin{array}{c}
\ln \mu_{k}^{1}=(-8704) / T+0.5 * \ln V+10 \\
Y \sim N\left(\mu_{k}^{1}\left(\Delta t_{k}^{1}\right),(0.002)^{2}\left(\Delta t_{k}^{1}\right)\right)
\end{array}\right.
$$

According to the constraint conditions in Reference [39] and the proposed optimization method, the optimization problem can be described as

$$
\begin{aligned}
& \max \frac{\left|M^{1}(\xi)\right|}{\left|M_{22}{ }^{1}(\xi)\right|} \\
& \text { s.t. } \quad\left\{\begin{array}{c}
C_{1}{ }^{1} \geq n^{1} \cdot C_{d}{ }^{1}+C_{0}{ }^{1} \cdot \sum_{l=1}^{L} m_{l}{ }^{1} \cdot \Delta t^{1} \\
S_{i 0}{ }^{1}<S_{i 1}{ }^{1}<S_{i 2}{ }^{1}<S_{i 3}{ }^{1} \cdots<S_{i L}{ }^{1}<S_{i m a x}{ }^{1} \\
m_{1}{ }^{1} \geq m_{2}{ }^{1} \geq \cdots \geq m_{l}{ }^{1} \geq 0, m_{1}{ }^{1} \geq \frac{m^{1}}{l^{1}} \\
n_{l}{ }^{1} \geq 3
\end{array} \quad \text { variables : } S_{i l}{ }^{1}, n_{l}{ }^{1}, m_{l}{ }^{1}\right.
\end{aligned}
$$

In Equation (31), $i=1,2 ; l=1,2,3,4$; the specific values of $C_{1}{ }^{1}, C_{d}{ }^{1}, C_{0}{ }^{1}, \Delta t$ and the values range of $S_{1}{ }^{1}, S_{2}{ }^{1}$ are shown in Table 16:

Table 16. Test cost and stress range.

\begin{tabular}{cccccc}
\hline$C_{1}^{1}$ (yuan) & $C_{d}^{1}$ (yuan) & $C_{0}^{1}$ (yuan) & $\Delta \mathbf{t}(\mathbf{h})$ & $\mathbf{T}(\mathbf{K})$ & $\mathbf{V ~ ( V )}$ \\
\hline 150,000 & 3000 & 200 & 1 & $313.15-383.15$ & $5-15$ \\
\hline
\end{tabular}

The proposed method is used to optimize the test scheme of the product in reference [39], and the objective function value of the scheme under $D$-optimality is calculated. The results are shown in Table 17.

Table 17. Optimization results.

\begin{tabular}{cccccc}
\hline $\boldsymbol{n}^{1}$ & $\mathbf{T}$ & $\mathbf{V}$ & $\boldsymbol{m}^{1}$ & $\left|\boldsymbol{M}^{1}(\boldsymbol{\xi})\right| /\left|\boldsymbol{M}_{22}^{1}(\boldsymbol{\xi})\right|$ & $\operatorname{det}|\boldsymbol{M}(\boldsymbol{\xi})|$ \\
\hline$[6666]$ & {$[368.15373 .15378 .15383 .15]$} & {$[11121314]$} & {$[99979797]$} & 742411.69 & $6.54 * 10^{13}$ \\
\hline
\end{tabular}

The optimal scheme under D-optimality in Reference [39] is optimized under the optimization method proposed in this paper, and the results are shown in Table 18.

Table 18. Optimization results of the optimal scheme in Reference [39].

\begin{tabular}{cccccc}
\hline $\boldsymbol{n}^{1}$ & $\mathbf{T}$ & $\mathbf{V}$ & $\boldsymbol{m}^{1}$ & $\left|\boldsymbol{M}^{1}(\xi)\right| / \mid \boldsymbol{M}_{22}^{1}(\xi)$ & $\operatorname{det}|\boldsymbol{M}(\xi)|$ \\
\hline$[6666]$ & {$[353.15358 .15378 .15383 .15]$} & {$[10131415]$} & {$[1131127575]$} & 439902.93 & $8.02 * 10^{13}$ \\
\hline
\end{tabular}


Because parameter $A^{1}$ is one parameter, the parameter covariance matrix $M^{11}{ }_{A^{1}}(\xi)$ is the variance matrix of parameter $A^{1}$, and because $M^{11}{ }_{A^{1}}(\xi)$ and $M^{1}(\xi) / M_{22}{ }^{1}(\xi)$ are inverse matrices, the determinant value of the variance matrix of parameter $A^{1}$ and $\left|M^{1}(\xi)\right| /\left|M_{22}{ }^{1}(\xi)\right|$ are reciprocal. Similarly, the determinant value of parameter covariance matrix and $\operatorname{det}\left|M^{1}(\xi)\right|$ are reciprocal. By comparing the values of $\left|M^{1}(\xi)\right| /\left|M_{22}{ }^{1}(\xi)\right|$ and $\operatorname{det}\left|M^{1}(\xi)\right|$ in Tables 17 and 18, we can get that the determinant value of variance matrix of the parameters of the test scheme optimized by the proposed method is 0.59 times of that under $D$-optimality, while the determinant value of covariance matrix of all parameters is 1.22 times of that under $D$-optimality. According to the comparison results, it can be concluded that the proposed optimization method can not only effectively improve the evaluation accuracy of main parameters, but also ensure the evaluation accuracy of non-main parameters in the optimization design of SSADT with multi stress, so as to solve the problem of low evaluation accuracy of important model parameters in the optimization design of SSADT with multi stress.

\section{Sensitivity Analysis}

At the beginning of the accelerated degradation test scheme design, the parameter $\gamma_{0}, \gamma_{1}, \gamma_{2}, \sigma$ in the stress model is usually obtained based on prior data or engineering experience, or estimated by a small number of grope tests. However, there are usually some deviations between these values and the true values. Therefore, the sensitivity of the model parameters to the test scheme is discussed and analyzed.

Assuming that the true value of model parameters is the initial value used in the calculation example, other conditions are consistent with the example, and the influence of parameter deviation on the optimization result of test scheme is analyzed. The deviation levels of each model parameter are $10 \%, 0 \%$, and $-10 \%$, respectively, and there are 81 combinations of deviation of the four parameters. In order to reduce the amount of calculation and make the selected deviation combination representative, orthogonal test $L_{9}\left(3^{4}\right)$ is used to conduct deviation test, and RV function is selected as the evaluation basis. The expression is shown in Equation (32) and the calculation results are shown in Table 19.

$$
R V=\left|\frac{\left|M^{11}(\xi)\right|-\left|M^{11}(\xi)\right|^{*}}{\left|M^{11}(\xi)\right|}\right| \times 100 \%
$$

where $\left|M^{11}(\xi)\right|$ is the objective function value obtained when the model parameters are constant; $\left|M^{11}(\xi)\right|^{*}$ is the objective function value obtained after the change of model parameters.

Table 19. Optimization results under different combinations of model parameter deviations.

\begin{tabular}{cccccccccc}
\hline$\gamma_{0}$ & $\gamma_{1}$ & $\gamma_{2}$ & $\sigma$ & $n$ & $S_{1}$ & $S_{2}$ & $\Delta t$ & $\boldsymbol{m}$ \\
\hline $10 \%$ & $10 \%$ & $10 \%$ & $10 \%$ & {$[5555]$} & {$[800850900950]$} & {$[6000650070007500]$} & 10 & {$[66626161]$} & $80.6 \%$ \\
$10 \%$ & 0 & 0 & 0 & {$[5555]$} & {$[800850900950]$} & {$[6000650070007500]$} & 10 & {$[66626161]$} & $46.9 \%$ \\
$10 \%$ & $-10 \%$ & $-10 \%$ & $-10 \%$ & {$[5555]$} & {$[800850900950]$} & {$[6000650070007500]$} & 10 & {$[66626161]$} & $93.6 \%$ \\
0 & $10 \%$ & 0 & $-10 \%$ & {$[5555]$} & {$[800850900950]$} & {$[6000650070007500]$} & 10 & {$[66626161]$} & $11.1 \%$ \\
0 & 0 & $-10 \%$ & $10 \%$ & {$[5555]$} & {$[800850900950]$} & {$[6000650070007500]$} & 10 & {$[66626161]$} & $47.0 \%$ \\
0 & $-10 \%$ & $10 \%$ & 0 & {$[5555]$} & {$[800850900950]$} & {$[6000650070007500]$} & 10 & {$[66626161]$} & $59.3 \%$ \\
$-10 \%$ & $10 \%$ & $-10 \%$ & 0 & {$[5555]$} & {$[800850900950]$} & {$[6000650070007500]$} & 10 & {$[66626161]$} & $32.2 \%$ \\
$-10 \%$ & 0 & $-10 \%$ & $-10 \%$ & {$[5555]$} & {$[800850900950]$} & {$[6000650070007500]$} & 10 & {$[66626161]$} & $25.3 \%$ \\
$-10 \%$ & $-10 \%$ & 0 & $10 \%$ & {$[5555]$} & {$[800850900950]$} & {$[6000650070007500]$} & 10 & {$[66626161]$} & $37.0 \%$ \\
\hline
\end{tabular}

From the results in the Table 19 that the scheme obtained by the optimization method is not sensitive to the variation of model parameters' deviation $(10 \%, 0,-10 \%)$, indicating that the method has good stability. From Table 19, the variation of parameters $\gamma_{0}$ and $\gamma_{1}$ has great influence on the deviation of the test scheme, especially when the parameters of $\gamma_{0}$ and $\gamma_{1}$ fluctuate at the same time, thus the influence is obvious. This is because the method proposed in the calculation example is based on the parameters for subsequent optimization. 
Therefore, in order to reduce the evaluation error of the subsequent parameters, the value range of the main parameters of the model should be as small as possible before the test, so as to ensure that the optimized test scheme can obtain higher accuracy.

\section{Conclusions}

1. In this paper, under the constraint of the test cost and the actual range of test variables, taking the test stress levels $S_{i l}$, sample size $n_{l}$ and inspection times $m_{l}$ as optimization variables, the optimization design of multi stress step accelerated degradation test scheme for a motorized spindle is carried out. When the objective function is established, the exhaustive method is used to solve the set of alternatives to avoid the wrong optimal solution caused by improper setting of the initial conditions of the optimization algorithm. By solving all possible combinations of stress levels under different sample sizes, it is concluded that the optimal combination of stress levels with different quantities of samples is the same under the constraint conditions.

2. The drift Brownian motion is used to model the accelerated degradation process of motorized spindle under multiple stresses. By selecting the main test stress and inspection indexes of the motorized spindle, the minimum estimation variance of main stress affecting the motorized spindle is taken as the optimization objective (i.e., $\min \left(\operatorname{det}\left(\left|M^{11}(\widetilde{\xi})\right|\right)\right)$ ), the optimization algorithm steps and detailed flow chart are given. Taking the accelerated degradation test of motorized spindle under two stresses as an example, the optimized test scheme is obtained, and compared with the traditional $D$-optimality optimization, the objective function value of the test scheme optimized by $D_{s}$-optimality is calculated under $D$-optimality, and the objective function value is only $18.5 \%$ lower than that of the optimal scheme optimized by $D$-optimality. Through an extended case study, it is concluded that when the proposed method is used to optimize the test scheme, the determinant value of the variance matrix of the main stress parameters is 0.59 times of that under $D$-optimality. It shows that this method can not only improve the evaluation accuracy of main stress parameters, but also ensure the evaluation accuracy of other stress parameters.

3. By analyzing the influence of the initial model parameter deviation on the optimization results, it is concluded that the optimization design method proposed in this paper has good stability. Since this method is based on the optimization criteria of model parameters, the range of main model parameters should be as small as possible before the test, so as to improve the accuracy of reliability evaluation of the optimized accelerated test scheme.

Author Contributions: Conceptualization, H.Z., Z.Y.; methodology, H.Z. and C.C.; software, H.Z.; validation, H.Z., C.C. and H.T.; investigation, X.L.; writing—original draft preparation, H.Z. and C.C.; writing-review and editing, H.Z., H.T. and S.L.; funding acquisition, H.Z. and C.C. All authors have read and agreed to the published version of the manuscript.

Funding: This work was supported by the National Natural Science Foundation of China under grant numbers 51975249 and 51675227; the Key Research and Development Plan of Jilin Province under grant numbers 20180201007GX and 20190302017GX; the Jilin Industry Innovation Project under grant number 2019C037-1; the Jilin Scientific Project of the Education Department under grant number JJKH20180133KJ; and the Fundamental Research Funds for the Central Universities.

Institutional Review Board Statement: Not applicable.

Informed Consent Statement: Not applicable.

Acknowledgments: Our deepest gratitude goes to the editors and reviewers for their constructive suggestions on the paper. We also thank the authors of the references, whose work contributed greatly to the completion of this paper.

Conflicts of Interest: The authors declare no conflict of interest. 


\section{References}

1. Yang, Z.J.; Chen, C.H.; Chen, F.; Li, G.F. Progress in the Research of Reliability Technology of Machine Tools. J. Mech. Eng. 2013, 49, 130-139. [CrossRef]

2. Luis, A.E.; William, Q.M. A Review of Accelerated Test Models. Stat. Sci. 2006, 21, 552-577.

3. Nelson, W. Analysis of performance degradation data from accelerated tests. IEEE Trans. Reliab. 1981, 30, 149-154. [CrossRef]

4. Nelson, W. Accelerated Testing: Statistical Methods, Test Plans, and Data Analysis, 1st ed.; John Wiley Press: New York, NY, USA, 1990; pp. 79-120.

5. Yang, G. Optimum degradation tests for comparison of products. IEEE Trans. Reliab. 2012, 61, 220-226. [CrossRef]

6. Chernoff. Optimal Accelerated Life Designs For Estimation. Technometrics 1962, 4, 381-409. [CrossRef]

7. Nelson, W.; Kielpinski, T.J. Theory for optimum censored accelerated life testing for normal and lognormal life distributions. Technometrics 1976, 18, 105-114. [CrossRef]

8. Nelson, W.; Meeker, W.Q. Theory for optimum accelerated censored life testing for Weibull and extreme value life Distributions. Technometrics 1978, 20, 171-177. [CrossRef]

9. Kielpinski, T.J.; Nelson, W. Optimum censored accelerated lifetesting for normal and lognormal life distributions. IEEE Trans. Reliab. 1975, 24, 310-320. [CrossRef]

10. Meeker, W.Q.; Nelson, W. Optimum accelerated censored lifetesting for Weibull and extreme value life distributions. IEEE Trans. Reliab. 1975, 24, 321-332. [CrossRef]

11. Meeker, W.Q. A comparison of accelerated life test plans for Weibull and lognormal distributions and type I censoring. Technometrics 1984, 26, 157-171. [CrossRef]

12. Park, J.W.; Yum, B.J. Optimal design of accelerated life tests with two stresses. Naval Res. Logist. 1996, 43, 863-884. [CrossRef]

13. Elsayed, E.A.; Zhang, H. Design of PH-based accelerated life testing plans under multiple-stress-type. Relia. Eng. Syst. Saf. 2007, 92, 286-292. [CrossRef]

14. Hu, C.H.; Lee, M.Y.; Tang, J. Optimal Step-stress accelerated degradation test for Wiener degradation process under constraints. Eur. J. Oper. Res. 2015, 241, 412-421. [CrossRef]

15. Lim, H.S.; Yum, B.J. Optimal design of accelerated degradation tests based on Wiener process models. J. Appl. Stat. 2011, 38, 309-325. [CrossRef]

16. Ge, Z.Z.; Li, X.Y.; Zhang, J.R.; Jiang, T.M. Planning of Step-Stress Accelerated Degradation Test with Stress Optimization. Adv. Mater. Res. 2010, 118-120, 404-408. [CrossRef]

17. Tsai, T.R.; Sung, W.Y.; Lio, Y.L.; Shing, I.C.; Lu, J.C. Optimal Two-Variable Accelerated Degradation Test Plan for Gamma Degradation Processes. IEEE Trans. Reliab. 2016, 65, 459-468. [CrossRef]

18. Tung, H.P.; Tseng, S.T. Planning gamma accelerated degradation tests with two accelerating variables. Naval Res. Logist. 2019, 66, 439-447. [CrossRef]

19. Mosavebi, O.E.; Shemehsavar, S. Optimal Design for Accelerated Degradation Test Based on D-Optimality. Iran. J. Sci. Technol. Trans. A Sci. 2018, 43, 1811-1818.

20. Sung, S.I.; Yum, B.J. Optimal design of step-stress accelerated degradation tests based on the Wiener degradation process. Qual. Technol. Quant. Manag. 2016, 13, 367-393. [CrossRef]

21. Li, X.Y.; Hu, H.Y.; Li, R.Q.; Liu, L. Bayesian optimal design of sequential stress accelerated degradation testing. In Proceedings of the 2016 Prognostics and System Health Management Conference (PHM-Chengdu), Chengdu, China, 19-21 October 2016; pp. 1-6.

22. Wang, Y.S.; Zhang, C.H.; Zhang, S.F.; Chen, X.; Tan, Y.Y. Optimal design of constant stress accelerated degradation test plan with multiple stresses and multiple degradation measures. Proc. IMechE Part O J. Risk Reliab. 2015, 229, 83-93. [CrossRef]

23. Wang, Y.; Zhang, C.; Chen, X. Study of simulationbased optimal design for degradation test. Astronaut 2008, $29,380-384$.

24. Li, X.Y.; Hu, H.Q.; Sun, F.Q.; Kang, R. A Bayesian optimal design for sequential accelerated degradation testing. Entropy 2017, 19, 325. [CrossRef]

25. Wang, H.; Zhao, Y.; Ma, X.B.; Wang, H.Y. Optimal design of constant-stress accelerated degradation tests using the M-optimality criterion. Relia. Eng. Syst. Saf. 2017, 164, 45-54. [CrossRef]

26. Zhao, X.J.; Xu, J.Y.; Liu, B. Accelerated Degradation Tests Planning With Competing Failure Modes. IEEE Trans. Reliab. 2018, 67, 142-155. [CrossRef]

27. Yu, Y.; Hu, C.H.; Si, X.S.; Zhang, J.X. Modified Bayesian D-Optimality for Accelerated Degradation Test Design With Model Uncertainty. IEEE Access 2019, 7, 42181-42189. [CrossRef]

28. Jiang, P.H.; Wang, B.X.; Wang, X.F.; Qin, S.D. Optimal plan for Wiener constant-stress accelerated degradation model. Appl. Math. Modell. 2020, 84, 191-201. [CrossRef]

29. Ma, Z.H.; Liao, H.T.; Ji, H.; Wang, S.P.; Yin, F.L.; Nie, S.L. Optimal design of hybrid accelerated test based on the Inverse Gaussian process model. Relia. Eng. Syst. Saf. 2021, 210, 107509-107519. [CrossRef]

30. Wang, Y.S.; Chen, X.; Tan, Y.Y. Optimal Design of Step-stress Accelerated Degradation Test with Multiple Stresses and Multiple Degradation Measures. Qual. Reliab. Eng. Int. 2017, 33, 1655-1668. [CrossRef]

31. Chen, W.H.; Liu, J.; Gao, L.; Pan, J.; Lu, X.B. Step-stress accelerated degradation test modeling and statistical analysis methods. Chin. J. Mech. Eng. 2013, 26, 1154-1159. [CrossRef]

32. Liu, R.Y.; Mao, S.S. Optimum Step-stress Plans for Accelerated Life Testing. Chin. J. Appl. Probab. 2002, 18, 34-42.

33. Wei, Z.J. Probability Theory and Mathematical Statistics, 2nd ed.; China Statistics Press: Beijing, China, 2013; pp. $230-261$. 
34. Li, X.X. Research on Multiple-Stress Reliability Accelerated Model of Spindle System in Machining Center. Ph.D. Thesis, Jilin University, Jilin, China, 2018.

35. Mao, S.S. Accelerated model of accelerated life test. Qual. Reliab. 2003, 2, 15-18.

36. Fang, B.R.; Zhou, J.M.; Li, Y.M. Matrix Theory, 2nd ed.; Tsinghua University Press: Beijing, China, 2013 ; pp. 112-158.

37. Zhao, H.X.; Yang, Z.J.; Chen, C.H.; Tian, H.L.; Chen, L.; Ying, J.; Jia, X. Development of Reliability Test System Based on Working Principle and Fault Analysis of Motorized Spindle. In Proceedings of the 2018 3rd International Conference on System Reliability and Safety (ICSRS), Barcelona, Spain, 22-25 November 2018; pp. 299-307.

38. Cai, Z.Y.; Chen, Y.X.; Zhang, Z.M.; Xiang, H.C. Reliability assessment method of nonlinear step-stress accelerated degradation data. J. Beijing Univ. Aeronaut. Astronaut. 2016, 42, 576-582.

39. Ge, Z.Z.; Jiang, T.M.; Han, S.H.; Li, X.Y. Design of accelerated degradation testing with multiple stresses based on D optimality. Syst. Eng. Electron. Technol. 2012, 34, 846-853. 